\title{
Light dilaton in a metastable vacuum
}

\author{
Daniel Elander $\odot,{ }^{1}$ Maurizio Piai, ${ }^{2}$ and John Roughley $\odot^{2}$ \\ ${ }^{1}$ Laboratoire Charles Coulomb (L2C), University of Montpellier, CNRS, 34095 Montpellier, France \\ ${ }^{2}$ Department of Physics, College of Science, Swansea University, \\ Singleton Park, SA2 8PP Swansea, Wales, United Kingdom
}

(Received 26 November 2020; accepted 28 January 2021; published 17 February 2021; corrected 12 April 2021)

\begin{abstract}
We identify a parametrically light dilaton by studying the perturbations of metastable vacua along a branch of regular supergravity backgrounds that are dual to four-dimensional confining field theories. The branch includes also stable and unstable solutions. The former encompass, as a special case, the geometry proposed by Witten as a holographic model of confinement. The latter approach a supersymmetric solution, by enhancing a condensate in the dual field theory. A phase transition separates the space of stable backgrounds from the metastable ones. In proximity of the phase transition, one of the lightest scalar states inherits some of the properties of the dilaton, despite not being particularly light.
\end{abstract}

DOI: $10.1103 /$ PhysRevD.103.046009

\section{INTRODUCTION}

The Higgs particle [1,2] might originate as a composite dilaton in a new strongly coupled theory. The literature on the effective field theory description of the dilaton has an ancient origin [3,4]. It has been invoked in the context of dynamical electroweak symmetry breaking [5-7], of extensions of the standard model [8-19], and in the interpretation of lattice data [20-30]. With the advent of gauge-gravity dualities [31-34], holographic models giving rise to a dilatonic state have been identified and studied both in the context of bottom-up [35-49] and top-down constructions derived from supergravity [50-54].

We pursue an alternative approach to the study of the dilaton, along the program announced in Ref. [55], which is inspired by Refs. [56-59], but is implemented within the rigorous framework of supergravity. We generalize the notion of proximity to the Breitenlohner-Freedman unitarity bound [60] — central to the arguments in Ref. [56]—in order to explore non-AdS (anti-de Sitter) backgrounds dual to confining theories, in regions of parameter space near tachyonic instabilities. We aim at ascertaining whether the spectrum of bound states includes a light dilaton.

In this paper we consider the toroidal compactifiction of the maximal supergravity theory in $D=7$ dimensions [61-65], that admits as a background solution the holographic description of confinement proposed by Witten

Published by the American Physical Society under the terms of the Creative Commons Attribution 4.0 International license. Further distribution of this work must maintain attribution to the author(s) and the published article's title, journal citation, and DOI. Funded by SCOAP. [66]—also used for phenomenological purposes by Sakai and Sugimoto $[67,68]$. We compare to the case of Romans theory [69]—see Refs. [55,70,71].

We focus on three branches of solutions: (i) regular solutions that include the Witten model and are interpreted as duals of four-dimensional confining theories, (ii) a class of supersymmetric solutions, and (iii) a branch of nonsupersymmetric solutions, that (locally) preserve sixdimensional Poincaré invariance but are badly singularthey do not even meet Gubser's criteria [72]. We compute the spectrum of fluctuations of the relevant scalar and spin-2 tensor states, using the gauge-invariant formalism of Refs. [73-77], hence extending the study of the spectra performed in Refs. [70,78]. We compare to the result of applying the probe approximation [79] in order to ascertain whether any of the scalar states have significant overlap with the trace of the stress-energy tensor and can hence be identified with an approximate dilaton.

In a region of parameter space the spectrum contains a parametrically light dilaton. We study the energetics along the three branches of solutions, by computing the free energy using holographic renormalization [80-82], and employing a simple scale-setting procedure to compare different backgrounds [83]. We present firm evidence of the existence of a phase transition in the gravity theory (see also Ref. [84]). The parametrically light dilaton emerges along the portion of the regular branch of solutions which contains metastable solutions, the lifetime of which is not known (but see Ref. [85]).

\section{THE GRAVITY MODEL}

We denote with hatted symbols quantities characterizing the theory in $D=7$ dimensions. The action, truncated to 


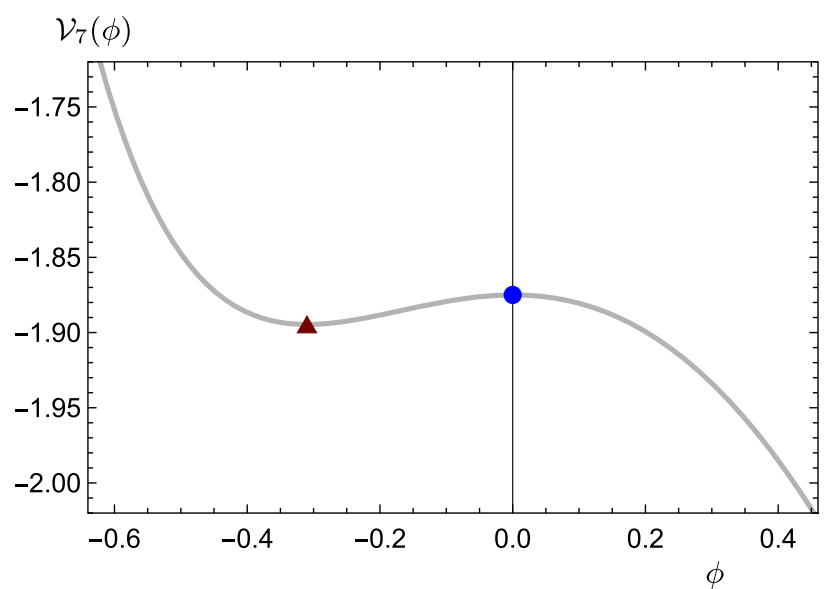

FIG. 1. The potential $\mathcal{V}_{7}(\phi)$ of the theory. The blue disk is the $\phi_{\mathrm{UV}}$ critical point, while the red triangle is the $\phi_{\mathrm{IR}}$ one.

retain the scalar $\phi$ coupled to gravity, is the following [79] (see also Refs. [62,63,70]):

$$
\mathcal{S}_{7}=\int \mathrm{d}^{7} x \sqrt{-\hat{g}_{7}}\left[\frac{\mathcal{R}_{7}}{4}-\frac{\hat{g}^{\hat{M} \hat{N}}}{4} \partial_{\hat{M}} \phi \partial_{\hat{N}} \phi-\mathcal{V}_{7}\right],
$$

where the potential (see Fig. 1) is

$$
\mathcal{V}_{7}=\frac{1}{8} e^{-\frac{8}{\sqrt{5}} \phi}-e^{-\frac{3}{\sqrt{5}} \phi}-e^{\frac{2}{\sqrt{5}} \phi} .
$$

This potential admits two critical points. The one with $\phi=\phi_{\mathrm{UV}}=0$ will play a central role in this paper, as it corresponds to a UV fixed point in the dual field theory. It yields $\mathcal{V}_{7}\left(\phi_{\mathrm{UV}}\right)=-\frac{15}{8}$. Another critical point of $\mathcal{V}_{7}$ has $\phi_{\mathrm{IR}}=-\frac{1}{\sqrt{5}} \log (2)$, for which $\mathcal{V}_{7}\left(\phi_{\mathrm{IR}}\right)=-\frac{5}{2^{7 / 5}}$.

Following the notation in Refs. [70,79], we reduce to $D=5$ dimensions by adopting the following ansatz:

$$
\mathrm{d} s_{7}^{2}=e^{-2 \chi} \mathrm{d} s_{5}^{2}+e^{3 \chi-2 \omega} \mathrm{d} \eta^{2}+e^{3 \chi+2 \omega} \mathrm{d} \zeta^{2},
$$

where the metric $\mathrm{d} s_{5}^{2}$ takes the domain wall form

$$
\mathrm{d} s_{5}^{2}=e^{2 A(r)} \mathrm{d} x_{1,3}^{2}+\mathrm{d} r^{2},
$$

and the background profiles $\phi(r), \chi(r), \omega(r)$, and $A(r)$ depend only on the radial coordinate $r$. The angles $0 \leq \eta$, $\zeta<2 \pi$ parametrize a torus. We apply the change of variables $\mathrm{d} \rho=e^{-\chi} \mathrm{d} r$. The domain-wall (DW) ansatz in $D=7$ dimensions is recovered by imposing the constraints $\omega=0$ and $\mathcal{A}=A-\chi=\frac{3}{5} A=\frac{3}{2} \chi$, and hence the $\mathrm{AdS}_{7}$ solution has $\partial_{\rho} \mathcal{A}=\frac{1}{2}, \partial_{\rho} \chi=\frac{1}{3}$, and $\partial_{\rho} A=\frac{5}{6}$. The bulk action in $D=5$ dimensions is the following:

$$
\mathcal{S}_{5}=\int \mathrm{d}^{5} x \sqrt{-g_{5}}\left[\frac{\mathcal{R}_{5}}{4}-\frac{g^{M N}}{2} G_{a b} \partial_{M} \Phi^{a} \partial_{N} \Phi^{b}-\mathcal{V}\right],
$$

where $\mathcal{V}=e^{-2 \chi} \mathcal{V}_{7}, \Phi^{a}=\{\phi, \omega, \chi\}$, and the sigma-model metric is $G_{a b}=\operatorname{diag}\left(\frac{1}{2}, 1, \frac{15}{4}\right)$. We verified that

$$
\mathcal{S}_{7}=\int \mathrm{d} \eta \mathrm{d} \zeta\left[\mathcal{S}_{5}+\int \mathrm{d}^{5} x \partial_{M}\left(\frac{1}{2} \sqrt{-g_{5}} g^{M N} \partial_{N} \chi\right)\right] .
$$

\section{CLASSES OF SOLUTIONS}

All the solutions of interest approach $\phi=\phi_{\mathrm{UV}}=0$ at large $\rho$. We write them as a power series of the small coordinate $z \equiv e^{-\rho / 2}$, as follows

$$
\begin{gathered}
\phi(z)=\phi_{2} z^{2}+\left(\phi_{4}-\frac{18 \phi_{2}^{2} \log (z)}{\sqrt{5}}\right) z^{4} \\
+\left(\frac{162}{5} \phi_{2}^{3} \log (z)-\frac{637 \phi_{2}^{3}}{30}-\frac{9 \phi_{2} \phi_{4}}{\sqrt{5}}\right) z^{6}+\cdots, \\
\omega(z)=\omega_{U}+\omega_{6} z^{6}+\cdots,
\end{gathered}
$$

$$
\begin{aligned}
\chi(z)= & \chi_{U}-\frac{2}{3} \log (z)-\frac{\phi_{2}^{2} z^{4}}{30}+\frac{2}{675}\left(\frac{675}{2} \chi_{6}-150 \omega_{6}\right. \\
& \left.+72 \sqrt{5} \phi_{2}^{3} \log (z)-6 \sqrt{5} \phi_{2}^{3}-20 \phi_{2} \phi_{4}\right) z^{6}+\cdots,
\end{aligned}
$$

$$
\begin{aligned}
A(z)= & A_{U}-\frac{5}{3} \log (z)-\frac{\phi_{2}^{2} z^{4}}{12}+\frac{1}{270}\left(\frac{135}{2} \chi_{6}-30 \omega_{6}\right. \\
& \left.+144 \sqrt{5} \phi_{2}^{3} \log (z)-12 \sqrt{5} \phi_{2}^{3}-40 \phi_{2} \phi_{4}\right) z^{6}+\cdots
\end{aligned}
$$

They are characterized by seven integration constants: $\phi_{2}, \phi_{4}, \omega_{U}, \omega_{6}, \chi_{U}, \chi_{6}$, and $A_{U}$. The DW solutions have $\omega_{U}=\omega_{6}=\chi_{6}=0$ and $\chi_{U}=\frac{2}{5} A_{U}$, leaving $A_{U}, \phi_{2}$ and $\phi_{4}$ as independent nontrivial free parameters. What we will call confining solutions have $\chi_{6}=0$.

We find it convenient to define a scale $\Lambda$ as follows [83]:

$$
\Lambda^{-1} \equiv \int_{\rho_{o}}^{\infty} \mathrm{d} \rho e^{\chi(\rho)-A(\rho)},
$$

with $\rho_{o}$ as the end of space. While other choices might be admissible, this has the advantage of being applicable to all the solutions of interest.

\section{A. SUSY solutions}

The supersymmetric (SUSY) DW solutions satisfy the following first-order differential equations:

$$
\partial_{\rho} \mathcal{A}=-\frac{2}{5} \mathcal{W}_{1}, \quad \partial_{\rho} \phi=2 \partial_{\phi} \mathcal{W}_{1}
$$

The superpotential $\mathcal{W}_{1} \equiv-\frac{1}{4} e^{-\frac{4}{\sqrt{5}} \phi}-e^{\frac{1}{\sqrt{5}} \phi}$ solves the defining equation $\mathcal{V}_{D}=\frac{1}{2} G^{\phi \phi}\left(\partial_{\phi} \mathcal{W}\right)^{2}-\frac{D-1}{D-2} \mathcal{W}^{2}$ for $D=7$. 
After performing the change of variables $\partial_{\rho} \equiv e^{-\frac{3 \phi}{2 \sqrt{5}}} \partial_{\tau}$, we find the exact solutions

$$
\phi(\tau)=\frac{4}{\sqrt{5}} \operatorname{arctanh}\left(e^{-2\left(\tau-\tau_{o}\right)}\right),
$$

with the warp factor given by

$$
\mathcal{A}(\tau)=\mathcal{A}_{o}+\frac{1}{10} \log \left[\cosh \left(\tau-\tau_{o}\right) \sinh ^{4}\left(\tau-\tau_{o}\right)\right],
$$

where $\mathcal{A}_{o}$ and $\tau_{o}$ are real integration constants.

The IR expansion of these solutions in terms of the radial coordinate $\rho$ and the new constants $\rho_{o}$ and $\mathcal{A}_{I}=\mathcal{A}_{0}+$ $\log \left(\frac{2}{5}\right)$ can be written explicitly in the following form

$$
\phi(\rho)=-\sqrt{5} \log \frac{2\left(\rho-\rho_{o}\right)}{5}+\frac{16\left(\rho-\rho_{o}\right)^{5}}{1875 \sqrt{5}}+\cdots,
$$

and

$$
\mathcal{A}(\rho)=\mathcal{A}_{I}+\log \left(\rho-\rho_{o}\right)+\frac{8\left(\rho-\rho_{o}\right)^{5}}{9375}+\cdots .
$$

Their holographic interpretation involves an operator of dimension $\Delta=4$ developing a vacuum expectation value in the dual field theory.

The conjugate superpotential entering the calculation of the free energy is known as a perturbative expansion:

$$
\mathcal{W}_{2}=-\frac{5}{4}-\frac{\phi^{2}}{4}+\frac{3 \phi^{3}}{4 \sqrt{5}} \log \left(\frac{\phi^{2}}{\kappa}\right)+\cdots,
$$

where $\kappa$ is scheme dependent.

\section{B. Singular DW solutions}

A class of singular DW solutions is characterized by the harmless $\mathcal{A}_{I}$, the end of space $\rho_{o}$, and the nontrivial $\phi_{5}$. As anticipated, these solutions are badly singular: their Ricci scalar tensor $\mathcal{R}_{7}$ diverges, and the potential is not bounded from above, violating the requirement from Ref. [72]. The IR expansion of solutions of this class reads as follows:

$$
\begin{aligned}
\phi(\rho)= & \frac{\sqrt{5}}{4} \log \frac{8}{5}\left(\rho-\rho_{o}\right)+\phi_{5}\left(\rho-\rho_{o}\right)^{5 / 8} \\
& +\frac{1}{135}\left(37 \sqrt{5} \phi_{5}^{2}-242^{3 / 4} \sqrt[4]{5}\right)\left(\rho-\rho_{o}\right)^{5 / 4}+\cdots, \\
\mathcal{A}(\rho)= & \mathcal{A}_{I}+\frac{\log \left(\rho-\rho_{o}\right)}{16}+\frac{4 \phi_{5}}{3 \sqrt{5}}\left(\rho-\rho_{o}\right)^{5 / 8} \\
& +\frac{1}{2700}\left(192 \times 10^{3 / 4}-2155 \phi_{5}^{2}\right)\left(\rho-\rho_{o}\right)^{5 / 4}+\cdots .
\end{aligned}
$$

\section{Confining solutions}

The regular solutions of this class obey the constraint $A=\frac{5}{2} \chi+\omega$. They depend on two harmless constants $\chi_{I}$ and $\omega_{I}$, besides $\rho_{o}$ and $\phi_{I}$. The IR expansion of these solutions reads as follows:

$$
\begin{aligned}
\phi(\rho)= & \phi_{I}-\frac{1}{2 \sqrt{5}}\left(\rho-\rho_{o}\right)^{2} e^{-\frac{8 \phi_{I}}{\sqrt{5}}}\left(-3 e^{\sqrt{5} \phi_{I}}+2 e^{2 \sqrt{5} \phi_{I}}+1\right) \\
& +\cdots, \\
\omega(\rho)= & \omega_{I}-\frac{\log \left(\rho-\rho_{o}\right)}{2} \\
& +\frac{1}{40}\left(\rho-\rho_{o}\right)^{2} e^{-\frac{8 \phi_{I}}{\sqrt{5}}}\left(8 e^{\sqrt{5} \phi_{I}}+8 e^{2 \sqrt{5} \phi_{I}}-1\right)+\cdots \\
\chi(\rho)= & \chi_{I}+\frac{\log \left(\rho-\rho_{o}\right)}{3} \\
& +\frac{1}{6000}\left(\rho-\rho_{o}\right)^{4} e^{-\frac{16 \phi_{I}}{\sqrt{5}}}\left(32 e^{\sqrt{5} \phi_{I}}-56 e^{2 \sqrt{5} \phi_{I}}\right. \\
& \left.+224 e^{3 \sqrt{5} \phi_{I}}+32 e^{4 \sqrt{5} \phi_{I}}-7\right)+\cdots
\end{aligned}
$$

We restrict attention to solutions flowing from the UV critical point, which requires $\phi_{I}>\phi_{\mathrm{IR}}$. The invariants $\mathcal{R}_{7}$,

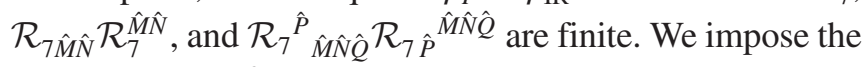
constraint $\omega_{I}=\frac{3}{2} \chi_{I}$ in order to avoid a conical singularity.

\section{GLUEBALL MASSES}

We compute the spectrum of fluctuations of the fivedimensional theory, by employing the gauge-invariant formalism developed in Refs. [73-77]. We introduce the IR regulator $\rho_{1}$ with $\rho_{o}<\rho_{1}$, and the UV regulator $\rho_{2}$. The physical results are recovered in the limits $\rho_{1} \rightarrow \rho_{o}$ and $\rho_{2} \rightarrow+\infty$ (see Refs. [70,71,77]). The scalar fluctuations are written as the gauge invariant combinations

$$
\mathfrak{a}^{a}(M, \rho) \equiv \varphi^{a}(M, \rho)-\frac{\partial_{\rho} \Phi^{a}(\rho)}{6 \partial_{\rho} A(\rho)} h(M, \rho),
$$

where $M$ is the mass in the dual theory, $\varphi^{a}$ are fluctuations of the scalars $\Phi^{a}$ and $h$ of the trace of the four-dimensional portion of the metric. They obey the following linearized equations and boundary conditions:

$$
0=\left[e^{\chi} \partial_{\rho}\left(e^{-\chi} \partial_{\rho}\right)+\left(4 \partial_{\rho} A\right) \partial_{\rho}+e^{2 \chi-2 A} M^{2}\right] \mathfrak{a}^{a}-e^{2 \chi} \chi^{a}{ }_{c} \mathfrak{a}^{c},
$$

$$
\begin{aligned}
0= & e^{-2 \chi} \partial_{\rho} \Phi^{c} \partial_{\rho} \Phi^{d} G_{d b} \partial_{\rho} \mathfrak{a}^{b}+\left[\frac{3 \partial_{\rho} A}{2} e^{-2 A} M^{2} \delta^{c}{ }_{b}\right. \\
& \left.-\partial_{\rho} \Phi^{c}\left(\frac{4 \mathcal{V}}{3 \partial_{\rho} A} \partial_{\rho} \Phi^{d} G_{d b}+\frac{\partial \mathcal{V}}{\partial \Phi^{b}}\right)\right]\left.\mathfrak{a}^{b}\right|_{\rho_{i}}
\end{aligned}
$$




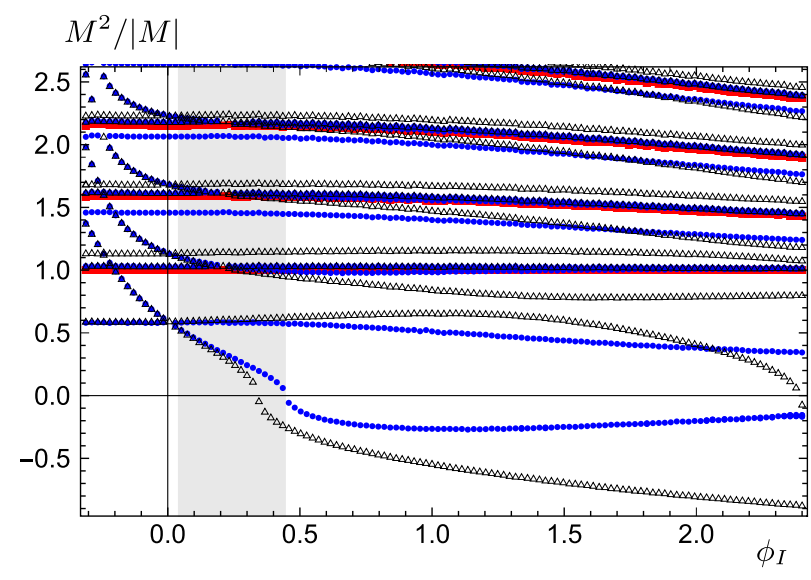

FIG. 2. The spectra of scalar masses $M$ as a function of the parameter $\phi_{I}$ along the confining branch of solutions, normalized in units of the lightest tensor mass, and using regulators $\rho_{1}=$ $10^{-4}$ and $\rho_{2}=12$. The (red) squares represent the spin- 2 tensor modes, the (blue) disks are the scalar, gauge invariant fluctuations originating from $\phi, \chi$, and $\omega$. The (black) triangles do not represent an additional set of states: they denote the same scalars, but computed in the probe approximation-neglecting the fluctuation of the background metric. The shading denotes the stable (leftmost white), metastable (gray), and unstable (rightmost white) backgrounds. We verified that our choices for the two regulators were sufficiently close to the physical limits $\left(\rho_{1} \rightarrow \rho_{o}\right.$, $\rho_{2} \rightarrow \infty$ ) to avoid discernible cutoff effects.

where in all these expressions the quantities $A, \Phi^{a}$, and $\mathcal{V}$ are evaluated on the background, and

$$
\begin{aligned}
\chi^{a}{ }_{c} \equiv & \partial_{c}\left[G^{a b} \frac{\partial \mathcal{V}}{\partial \Phi^{b}}\right] \\
& +\frac{4}{3 \partial_{\rho} A}\left[\partial_{\rho} \Phi^{a} \frac{\partial \mathcal{V}}{\partial \Phi^{c}}+G^{a b} \frac{\partial \mathcal{V}}{\partial \Phi^{b}} \partial_{\rho} \Phi^{d} G_{d c}\right] \\
& +\frac{16 \mathcal{V}}{9\left(\partial_{\rho} A\right)^{2}} \partial_{\rho} \Phi^{a} \partial_{\rho} \Phi^{b} G_{b c} .
\end{aligned}
$$

The gauge invariant spin-2 tensor fluctuations obey the linearized equation

$$
0=\left[\partial_{\rho}^{2}+\left(4 \partial_{\rho} A-\partial_{\rho} \chi\right) \partial_{\rho}+e^{2 \chi-2 A} M^{2}\right] \mathbf{e}_{\nu}^{\mu},
$$

and Neumann boundary conditions $\left.\partial_{\rho} \mathrm{e}^{\mu}\right|_{\rho_{i}}=0$.

The probe approximation for the scalars is defined by ignoring the term proportional to $h$ in Eq. (24). According to the dictionary of gauge-gravity dualities, $h$ is the bulk field associated with the trace of the stress-energy tensor, which is the field theory operator associated with dilatation, and sourcing the dilaton. Hence, this approximation holds for scalar bound states that decouple from the dilatation operator, and cannot be interpreted as a dilaton. The equations for the scalar fluctuations greatly simplify, as only the first term in Eq. (26) survives, and the boundary conditions reduce to Dirichlet. Note that the probe approximation is used solely as a diagnostic tool to identify scalar states which mix nontrivially with the dilaton.

In Fig. 2, we show the spectra of tensors and scalars, compared to the probe approximation, normalized to the lightest spin-2 fluctuation. For $\phi_{I}<0$ the scalars agree with Ref. [70]. The new results for $\phi_{I}>0$ show that one of the scalars becomes parametrically light, and eventually tachyonic, for positive $\phi_{I}$. The mass vanishes exactly at some finite value of $\phi_{I}$, for which the background geometry is still describing the dual of a confining field theory, in the presence of nonvanishing condensates. When this state is light, or tachyonic, the probe approximation does not capture it correctly, indicating that the state has a nontrivial component along $h$, and hence is sourced by the trace of the stress-energy tensor, as expected by a dilaton. We also notice that several of the heavy scalar states are not well captured by the probe approximation, showing that mixing effects with the dilaton are not restricted to the lightest states.

\section{FREE ENERGY}

To compute the free energy, we write explicitly the boundary terms of the theory in $D=7$ dimensions:

$\mathcal{S}=\mathcal{S}_{7}+\sum_{i=1,2}(-)^{i} \int \mathrm{d}^{4} x \mathrm{~d} \zeta \mathrm{d} \eta \sqrt{-\tilde{\hat{g}}}\left[\frac{K}{2}+\lambda_{i}\right]_{\rho=\rho_{i}}$,

where $\tilde{\hat{g}}$ denotes the determinant of the pullback of the induced metric, $K$ is the Gibbons-Hawking-York term and $\lambda_{i}$ are localized boundary potentials.

The potential terms are chosen according to the same prescription as in Ref. [55]: in the UV we replace $\lambda_{2}=\mathcal{W}_{2}$, which allows one to cancel all the divergences and perform the program of holographic renormalization [80-82], while in the IR we impose $\lambda_{1}=-\frac{3}{2} \partial_{\rho} A(\rho)$, in such a way that the variational problem be well defined in the presence of the IR boundary at $\rho=\rho_{1}$. The free energy density $\mathcal{F}$ is defined in terms of the complete on-shell action to be

$$
\int \mathrm{d}^{4} x \mathrm{~d} \zeta \mathrm{d} \eta \mathcal{F} \equiv-\lim _{\rho_{2} \rightarrow \infty} \lim _{\rho_{1} \rightarrow \rho_{o}} \mathcal{S}_{\text {on-shell }}
$$

By making use of the equations of motion we arrive at

$$
\mathcal{F}=-\left.\lim _{\rho_{2} \rightarrow \infty} e^{4 A-\chi}\left(\frac{3}{2} \partial_{\rho} A+\mathcal{W}_{2}\right)\right|_{\rho_{2}},
$$

which is identical to Eq. (5.22) of Ref. [55].

We make use of the UV expansions of the background solutions of interest. By replacing the UV expansions in Eqs. (7)-(10) into the form of the free energy density in Eq. (30), supplemented by the specific form of the superpotential $\mathcal{W}_{2}$ in Eq. (17), we arrive at the expression: 


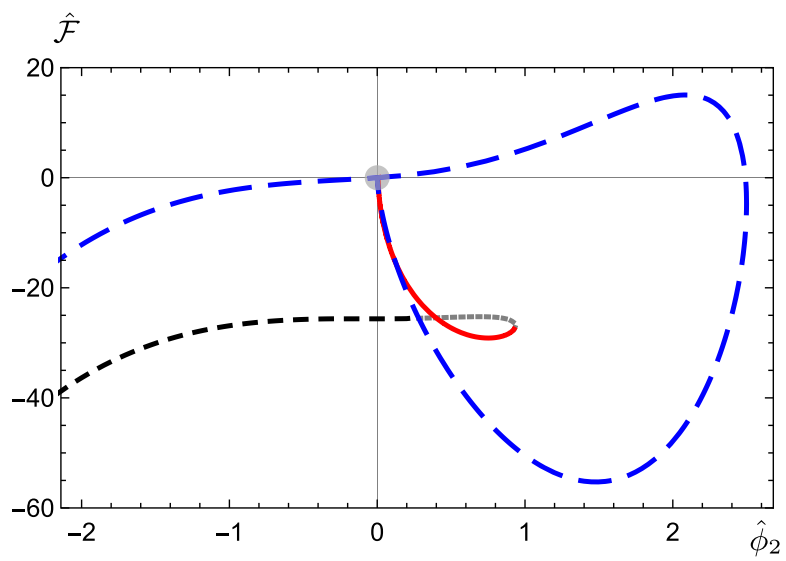

FIG. 3. The free energy density $\hat{\mathcal{F}}=\mathcal{F} \Lambda^{-6}$ as a function of the deformation parameter $\hat{\phi}_{2}=\phi_{2} \Lambda^{-2}$. The black (dashed), gray (short dashed), and red (solid) lines represent the stable, metastable and unstable portions of the confining branch of solutions. In blue (long dashed) we show the singular DW solutions. The SUSY solutions are represented by the gray disk at the origin of the plot.

$$
\begin{aligned}
\mathcal{F}= & -\frac{e^{4 A_{U}-\chi_{U}}}{120}\left(20 \phi_{2} \phi_{4}-135 \chi_{6}+60 \omega_{6}\right. \\
& \left.+12 \sqrt{5} \phi_{2}^{3}\left(-2+\frac{3}{2} \log \left(\frac{\phi_{2}^{2}}{\kappa}\right)\right)\right) .
\end{aligned}
$$

The divergence of the contribution to the free energy proportional to $\phi_{2}^{2}$ is canceled by $\mathcal{W}_{2}$. This implies that, as for the circle reduction of the Romans supergravity [55], the concavity theorems do not apply to $\mathcal{F}$. This expression still contains a residual scheme dependence in the logarithmic term. We set $\kappa=e^{-4 / 3}$, and hence our final expression for the free energy density is

$$
\begin{aligned}
\mathcal{F}= & -\frac{e^{4 A_{U}-\chi_{U}}}{120}\left(20 \phi_{2} \phi_{4}-135 \chi_{6}+60 \omega_{6}\right. \\
& \left.+18 \sqrt{5} \phi_{2}^{3} \log \left(\phi_{2}^{2}\right)\right) .
\end{aligned}
$$

We also remind the reader that $\chi_{6}=0$ in the background solutions of interest.

In Fig. 3 we show the free energy of the three classes of solutions, as a function of the deforming parameter $\hat{\phi}_{2} \equiv \phi_{2} \Lambda^{-2}$, and setting $A_{U}=0=\chi_{U}$. The parameters $\phi_{4}$ and $\omega_{6}$ are response functions, themselves determined nonlinearly, on each branch of solutions, by the choice of $\phi_{2}$. The SUSY solutions have $\mathcal{F}=0$.

The figure shows evidence of the existence of a firstorder phase transition. The confining solutions minimize $\hat{\mathcal{F}}$ for negative $\phi_{I}$. For $\phi_{I}>\phi_{I}^{c}$, with $\phi_{I}^{c} \simeq 0.039$ the critical value (corresponding to $\hat{\phi}_{2}^{c} \simeq 0.281$ ), the singular DW solutions have lower, finite free energy density $\hat{\mathcal{F}}$, so that the solutions along the confining branch are at best metastable when $\phi_{I}>\phi_{I}^{c}$, and eventually become unstable, with one of their fluctuations becoming tachyonic when $\phi_{I} \gtrsim 0.447$. Most interestingly, along the metastable branch, the lightest state becomes parametrically light, before becoming tachyonic (see Fig. 2). The probe approximation fails to capture correctly its mass squared when it is either small or negative. This eigenstate of the system is hence an admixture containing a significant contribution from the trace of the fluctuation of the metric-we interpret this finding as evidence that the state is approximately a dilaton.

\section{OUTLOOK}

We presented evidence of the emergence of a parametrically light dilatonic state along the metastable portion of a branch of regular backgrounds of the supergravity system in $D=7$ dimensions that yields also the Witten model, the first known holographic description of a four-dimensional confining theory [66]. Furthermore, the results of our analysis confirm, in the rigorous context of top-down holography, the expectations from Ref. [59] that along the stable portion of the regular branch a dilatonic state persists, but it is not parametrically light.

The metastable vacua, and the accompanying parametrically light dilatonic state, are new findings. Comparison with Ref. [55] indicates that this is a generic feature, which emerges in a broad class of theories. It would be interesting to discover examples in which the phase transition is weaker, and the spectrum along the stable branch exhibits a light approximate dilaton. It would also be useful to identify the requirements a supergravity theory must fulfil for such features to emerge.

\section{ACKNOWLEGMENTS}

We thank Alex Pomarol for useful discussions. The work of M.P. is supported in part by the STFC Consolidated Grants No. ST/P00055X/1 and No. ST/T000813/1. M. P. has received funding from the European Research Council (ERC) under the European Union's Horizon 2020 research and innovation programme, Grant Agreement No. 813942. J.R. is supported by STFC through the studentship ST/ R505158/1. 
[1] G. Aad et al. (ATLAS Collaboration), Phys. Lett. B 716, 1 (2012).

[2] S. Chatrchyan et al. (CMS Collaboration), Phys. Lett. B 716, 30 (2012).

[3] A. A. Migdal and M. A. Shifman, Phys. Lett. 114B, 445 (1982).

[4] S. Coleman, Aspects of Symmetry: Selected Erice Lectures (Cambridge University Press, Cambridge, England, 2010).

[5] C. N. Leung, S. T. Love, and W. A. Bardeen, Nucl. Phys. B273, 649 (1986).

[6] W. A. Bardeen, C. N. Leung, and S. T. Love, Phys. Rev. Lett. 56, 1230 (1986).

[7] K. Yamawaki, M. Bando, and K. I. Matumoto, Phys. Rev. Lett. 56, 1335 (1986).

[8] W. D. Goldberger, B. Grinstein, and W. Skiba, Phys. Rev. Lett. 100, 111802 (2008).

[9] D. K. Hong, S. D. H. Hsu, and F. Sannino, Phys. Lett. B 597, 89 (2004).

[10] D. D. Dietrich, F. Sannino, and K. Tuominen, Phys. Rev. D 72, 055001 (2005).

[11] M. Hashimoto and K. Yamawaki, Phys. Rev. D 83, 015008 (2011).

[12] T. Appelquist and Y. Bai, Phys. Rev. D 82, 071701 (2010).

[13] L. Vecchi, Phys. Rev. D 82, 076009 (2010).

[14] Z. Chacko and R. K. Mishra, Phys. Rev. D 87, 115006 (2013).

[15] B. Bellazzini, C. Csaki, J. Hubisz, J. Serra, and J. Terning, Eur. Phys. J. C 73, 2333 (2013).

[16] B. Bellazzini, C. Csaki, J. Hubisz, J. Serra, and J. Terning, Eur. Phys. J. C 74, 2790 (2014).

[17] T. Abe, R. Kitano, Y. Konishi, K. Y. Oda, J. Sato, and S. Sugiyama, Phys. Rev. D 86, 115016 (2012).

[18] E. Eichten, K. Lane, and A. Martin, arXiv:1210.5462.

[19] P. Hernandez-Leon and L. Merlo, Phys. Rev. D 96, 075008 (2017).

[20] S. Matsuzaki and K. Yamawaki, Phys. Rev. Lett. 113, 082002 (2014).

[21] M. Golterman and Y. Shamir, Phys. Rev. D 94, 054502 (2016).

[22] A. Kasai, K. I. Okumura, and H. Suzuki, arXiv:1609.02264.

[23] M. Hansen, K. Langaeble, and F. Sannino, Phys. Rev. D 95, 036005 (2017).

[24] M. Golterman and Y. Shamir, Phys. Rev. D 95, 016003 (2017).

[25] T. Appelquist, J. Ingoldby, and M. Piai, J. High Energy Phys. 07 (2017) 035.

[26] T. Appelquist, J. Ingoldby, and M. Piai, J. High Energy Phys. 03 (2018) 039.

[27] M. Golterman and Y. Shamir, Phys. Rev. D 98, 056025 (2018).

[28] O. Cata and C. Muller, Nucl. Phys. B952, 114938 (2020).

[29] T. Appelquist, J. Ingoldby, and M. Piai, Phys. Rev. D 101, 075025 (2020).

[30] O. Catà, R. J. Crewther, and L. C. Tunstall, Phys. Rev. D 100, 095007 (2019).

[31] J. M. Maldacena, Int. J. Theor. Phys. 38, 1113 (1999); Adv. Theor. Math. Phys. 2, 231 (1998).

[32] S. S. Gubser, I. R. Klebanov, and A. M. Polyakov, Phys. Lett. B 428, 105 (1998).

[33] E. Witten, Adv. Theor. Math. Phys. 2, 253 (1998).
[34] O. Aharony, S. S. Gubser, J. M. Maldacena, H. Ooguri, and Y. Oz, Phys. Rep. 323, 183 (2000).

[35] W. D. Goldberger and M. B. Wise, Phys. Rev. Lett. 83, 4922 (1999).

[36] O. DeWolfe, D. Z. Freedman, S. S. Gubser, and A. Karch, Phys. Rev. D 62, 046008 (2000).

[37] W. D. Goldberger and M. B. Wise, Phys. Lett. B 475, 275 (2000).

[38] C. Csaki, M. L. Graesser, and G. D. Kribs, Phys. Rev. D 63, 065002 (2001).

[39] N. Arkani-Hamed, M. Porrati, and L. Randall, J. High Energy Phys. 08 (2001) 017.

[40] R. Rattazzi and A. Zaffaroni, J. High Energy Phys. 04 (2001) 021.

[41] L. Kofman, J. Martin, and M. Peloso, Phys. Rev. D 70, 085015 (2004).

[42] D. Elander and M. Piai, Nucl. Phys. B864, 241 (2012).

[43] D. Kutasov, J. Lin, and A. Parnachev, Nucl. Phys. B863, 361 (2012).

[44] R. Lawrance and M. Piai, Int. J. Mod. Phys. A 28, 1350081 (2013).

[45] D. Elander and M. Piai, Nucl. Phys. B867, 779 (2013).

[46] M. Goykhman and A. Parnachev, Phys. Rev. D 87, 026007 (2013).

[47] N. Evans and K. Tuominen, Phys. Rev. D 87, 086003 (2013).

[48] E. Megias and O. Pujolas, J. High Energy Phys. 08 (2014) 081.

[49] D. Elander, R. Lawrance, and M. Piai, Nucl. Phys. B897, 583 (2015).

[50] D. Elander, C. Nunez, and M. Piai, Phys. Lett. B 686, 64 (2010).

[51] D. Elander and M. Piai, Nucl. Phys. B871, 164 (2013).

[52] D. Elander, Phys. Rev. D 91, 126012 (2015).

[53] D. Elander and M. Piai, Phys. Lett. B 772, 110 (2017).

[54] D. Elander and M. Piai, J. High Energy Phys. 06 (2017) 003.

[55] D. Elander, M. Piai, and J. Roughley, arXiv:2010.04100.

[56] D. B. Kaplan, J. W. Lee, D. T. Son, and M. A. Stephanov, Phys. Rev. D 80, 125005 (2009).

[57] V. Gorbenko, S. Rychkov, and B. Zan, J. High Energy Phys. 10 (2018) 108.

[58] V. Gorbenko, S. Rychkov, and B. Zan, SciPost Phys. 5, 050 (2018).

[59] A. Pomarol, O. Pujolas, and L. Salas, J. High Energy Phys. 10 (2019) 202.

[60] P. Breitenlohner and D. Z. Freedman, Ann. Phys. (N.Y.) 144, 249 (1982).

[61] H. Nastase, D. Vaman, and P. van Nieuwenhuizen, Phys. Lett. B 469, 96 (1999).

[62] M. Pernici, K. Pilch, and P. van Nieuwenhuizen, Phys. Lett. 143B, 103 (1984).

[63] M. Pernici, K. Pilch, P. van Nieuwenhuizen, and N.P. Warner, Nucl. Phys. B249, 381 (1985).

[64] H. Lu and C. N. Pope, Phys. Lett. B 467, 67 (1999).

[65] V. L. Campos, G. Ferretti, H. Larsson, D. Martelli, and B. E. W. Nilsson, J. High Energy Phys. 06 (2000) 023.

[66] E. Witten, Adv. Theor. Math. Phys. 2, 505 (1998).

[67] T. Sakai and S. Sugimoto, Prog. Theor. Phys. 113, 843 (2005). 
[68] T. Sakai and S. Sugimoto, Prog. Theor. Phys. 114, 1083 (2005).

[69] L. J. Romans, Nucl. Phys. B269, 691 (1986).

[70] D. Elander, A. F. Faedo, C. Hoyos, D. Mateos, and M. Piai, J. High Energy Phys. 05 (2014) 003.

[71] D. Elander, M. Piai, and J. Roughley, J. High Energy Phys. 02 (2019) 101.

[72] S. S. Gubser, Adv. Theor. Math. Phys. 4, 679 (2000).

[73] M. Bianchi, M. Prisco, and W. Mueck, J. High Energy Phys. 11 (2003) 052.

[74] M. Berg, M. Haack, and W. Mueck, Nucl. Phys. B736, 82 (2006).

[75] M. Berg, M. Haack, and W. Mueck, Nucl. Phys. B789, 1 (2008).

[76] D. Elander, J. High Energy Phys. 03 (2010) 114.

[77] D. Elander and M. Piai, J. High Energy Phys. 01 (2011) 026.

[78] R. C. Brower, S. D. Mathur, and C. I. Tan, Nucl. Phys. B587, 249 (2000).

[79] D. Elander, M. Piai, and J. Roughley, J. High Energy Phys. 06 (2020) 177.
[80] M. Bianchi, D. Z. Freedman, and K. Skenderis, Nucl. Phys. B631, 159 (2002).

[81] K. Skenderis, Classical Quant. Grav. 19, 5849 (2002).

[82] I. Papadimitriou and K. Skenderis, IRMA Lect. Math. Theor. Phys. 8, 73 (2005).

[83] C. Csaki, J. Erlich, T. J. Hollowood, and J. Terning, Phys. Rev. D 63, 065019 (2001).

[84] A. F. Faedo, M. Piai, and D. Schofield, Phys. Rev. D 89, 106001 (2014).

[85] F. Bigazzi, A. Caddeo, A. L. Cotrone, and A. Paredes, J. High Energy Phys. 12 (2020) 200.

Correction: Minor errors were found in Eq. (32) and the inline equation appearing before it and have been fixed. The fifth sentence that previously appeared after Eq. (32) has been deleted. The terms $\phi_{5}^{c}$ and $\hat{\mathcal{F}}$ have been deleted from the first parenthetical phrase appearing in the last paragraph of Sec. V. Figure 3 contained an error and has been replaced. 